\title{
Uroonkologie: Tabus brechen
}

\section{Onkologische Rehabilitation verbessert die Lebensqualität}

Das Prostatakarzinom ist der derzeit beim Mann am häufigsten diagnostizierte maligne Tumor. Nicht jedes Prostatakarzinom schränkt den Lebenskomfort oder die Lebensdauer vollkommen ein - für die Betroffenen ergeben sich neue Herausforderungen. Diese Herausforderungen rund um das Thema Uroonkologie und damit verbundene Tabus standen Mitte Mai beim 3. LEBENS. MEDizinischen Kongress - Pflege im Lebens.Med Zentrum Bad Erlach im Fokus.

Aufgrund der verbesserten Lebenserwartung und den medizinischen Fortschritten in der Urologie und Onkologie nimmt die Zahl der Menschen, die mit einer Krebserkrankung leben, zu. Rehabilitation als Teil eines uroonkologischen Gesamtkonzeptes gewinnt daher an Bedeutung. „Begleiterkrankungen bei den uroonkologischen Patienten, wie Depressivität, Distress, Müdigkeit oder Erschöpfung, werden bei dem evidenzbasierten und hochwirksamen Verfahren der Onkologischen Rehabilitation behandelt", stellte Univ.-Prof. Dr. Alexander Gaiger, Ärztlicher Leiter der Abteilung Onkologische Rehabilitation, Lebens. Med Zentrum Bad Erlach, fest. Allerdings nehmen derzeit nur etwa fünf Prozent der Betroffenen diese Möglichkeit in Anspruch, wie Pflegedienstleiter DGKP Jürgen Friedl, MSc und Organisator der Veranstaltung feststellte.

\section{Den Patienten abholen, wo er steht}

Auf die Ziele und Wirkung der psychosozialen Beratung ging DGKP Sonja Kofler, Stationsleitung im Lebens.Med Zentrum Bad Erlach, unter dem Titel „Pflege braucht mehr - Kommunikation und psychosoziale Beratung in der Uroonkologie“ ein. Ihr Appell: „Der Patient muss dort abgeholt werden, wo er steht und es soll nicht das durchgesetzt werden, was wir als Pflegefachkräfte als wichtig empfinden." So stellt auch die Ernährung bei uroonkologischen Patienten einen wesentlichen Teil der Lebensqualität dar - der allerdings etwa durch ernährungsrelevante Nebenwirkungen, die Appetitlosigkeit, einen veränderten Geruchs- und Geschmackssinn, Blähungen oder Durchfall, aber auch Gewichtszunahme und Mundtrockenheit vielfach beeinträchtigt sein kann und daher behutsamer Beratung und Begleitung bedarf. Mit praktischen Tipps können hier manche Schwierigkeiten bewältigt werden, wie Diätologin Mag. Angelika Beirer darstellte.

Schmerz, Inkontinenz und Uro-Stomata sind häufige Begleiterscheinungen bei uroonkologischen Erkrankungen. In beiden Fällen spielt die Pflege eine wichtige Rolle. „Schmerzmanagement ist Sache der Pflege“, erklärte Mag. (FH) Andrea Praher, Schmerzambulanz Med. Campus III, Kepler Universitätsklinikum Linz: „Es gibt kein Patentrezept, sehr wohl aber Mindestanforderungen bzw. Strategien. Jedenfalls ist Vorbeugung besser als Nachsorge." StomaTräger müssen gut versorgt werden, damit sie keine Einschränkungen haben und wieder gut ins Leben integriert werden. Dabei, so Dr. Mons Fischer und die Kontinenzund Stomaberaterinnen DGKP Susanne Rosner und DGKP Waltraud Ünal, wünschen sich die Stoma-Träger fachliche Beratung, Unauffälligkeit, Selbstversorgung/ Unabhängigkeit und einen einfachen $\mathrm{Zu}$ gang zu Versorgungsmaterialien.

\section{Soziale Systeme und Alltagsaktivitäten}

Für die sozialen Systeme, in denen sich der Krebserkrankte bewegt und die ihm Sicherheit bieten - wie Familie, Arbeitsplatz oder Freundeskreis - entstehen neue Herausforderungen. „Vor allem die Kinder

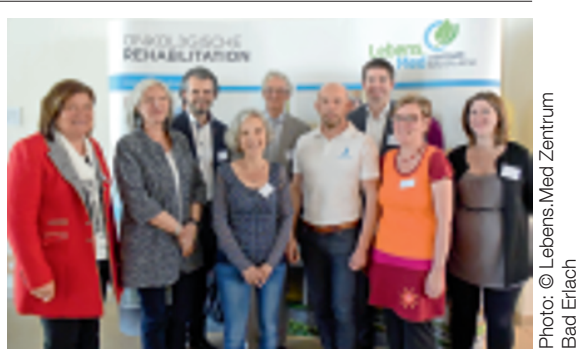

Expertenteam des 3. LEBENS.MEDizinischen Kongresses - Pflege im Fokus

sind häufig mit den weitreichenden Veränderungen durch die Diagnose Krebs eines Familienmitgliedes überfordert", so Mag. Andrea Keck, „und reagieren mit den unterschiedlichsten Verhaltensweisen." Mit einer möglichst unveränderten Beibehaltung des Alltagsablauf kann hier ein bisschen Normalität und Stabilität gesichert werden.

Sexualität, Intimität und Sport stellen in unterschiedlicher Intensität - für Gesunde einen normalen Bestandteil ihres Lebens dar. Im Falle einer Krebserkrankung rücken diese Aktivitäten oft in den Hintergrund. Sie behalten aber für den $\mathrm{Be}$ troffenen ihre Bedeutung. „Das Vorurteil, dass Sex für den Patienten nicht wichtig ist, muss ausgeräumt werden", forderte daher Dr. Lucia Ucsnik vom AKH Wien und wies auf die essentielle Rolle sexueller Gesundheit bei Heilung, Reintegration, Behandlung und Pflege hin. Eine dauerhafte Verbesserung der Leistungsfähigkeit in Sport und Bewegung sowie des Allgemeinempfindens könne auch bei Prostatakarzinom nur durch regelmäßiges Training erreicht werden, konstatierte Christian Seidl, Therapieleiter des Lebens. Med Zentrum Bad Erlach. Eine entsprechende professionelle Begleitung sei dafür empfehlenswert.

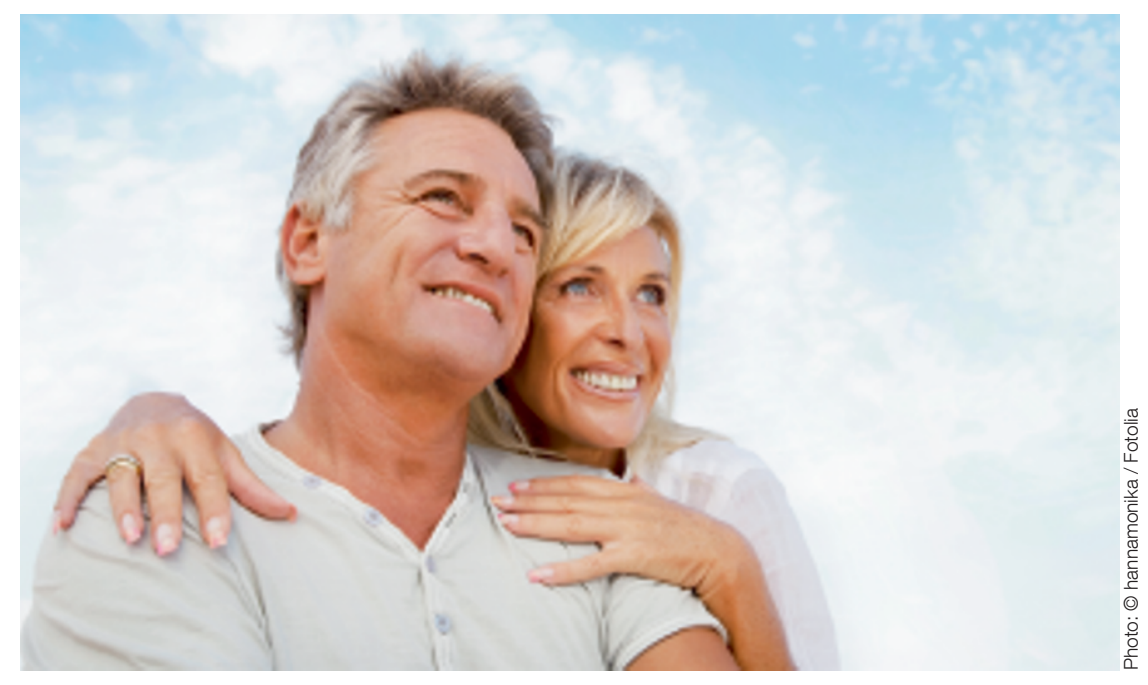

